\title{
Response to DARPA-SN-17-57 RFI
}

Title: "PICkLE" - Path to Iterative Confidence Level Evaluation

Technical point of contact:

Brian Nosek, Executive Director

Center for Open Science

210 Ridge McIntire Road, Suite 500

Charlottesville, VA 22903

434-806-6460

nosek@cos.io

July 2017

RFI questions addressed by this response:

The ideas described herein generally address the entire RFI and aim to identify and validate the State of the Art technologies, empirically describe their accuracy and resource demands, and compare the accuracy between evaluations using different data sources.

With this groundwork in place, exploration of algorithms to aggregate multiple credibility assessment techniques to achieve the highest accuracy at the lowest resource demand would provide hypotheses for future work and help identify key investments to maximize return on investment. 


\section{$\underline{\text { Technical Description }}$}

The credibility of evidence is important. This statement is as vapid as it is difficult to assess. There is no shortage of evidence or claims, but the tools to assess their credibility are immature, particularly at scale. There is enormous potential return on investment from a dedicated effort that (a) assesses existing methods of determining credibility, (b) fosters innovation in methods, and (c) conducts comparative analysis of accuracy versus resource cost.

It is possible to construct a framework for assessing credibility of research evidence. At the base would be high-throughput methods that provide rapid assessment at scale with known degree of uncertainty (accuracy). When greater confidence is needed, methods could be deployed that decrease uncertainty, but at a higher resource cost.

Optimization of decision-making would consider the accuracy-resource tradeoff for evidence acquisition and evaluation.

For example, consider this hypothetical table of methodologies available to evaluate claims from a particular research finding, paper, body of work, researcher, or research community. At the bottom are automated indicators that analyze available data and apply heuristics, algorithms, or machine learning to provide indicators of confidence. These can be applied at scale for comparatively low cost. The ceiling on their potential accuracy (as a marker for credibility of the research in question) is unknown. At the top

\begin{tabular}{|l|c|c|}
\hline $\begin{array}{l}\text { Confidence Assessment Method for } \\
\text { Claims }\end{array}$ & $\begin{array}{c}\text { Perceived } \\
\text { Accuracy }\end{array}$ & $\begin{array}{c}\text { Resource } \\
\text { Demand (\$, time) }\end{array}$ \\
\hline Repeated replications & 10 & 10 \\
\hline Single replication & 9 & 9 \\
\hline Meta-analysis of existing evidence & 8 & 8 \\
\hline Prediction market & 7 & 6 \\
\hline Surveys of experts & 6 & 5 \\
\hline Surveys of anyone & 5 & 3 \\
\hline $\begin{array}{l}\text { Distillation of public data from credible } \\
\text { sources }\end{array}$ & 4 & 2 \\
\hline $\begin{array}{l}\text { Automated indicators drawing on available } \\
\text { data (e.g., citation patterns, news coverage, } \\
\text { natural language assessment) }\end{array}$ & 3 & \\
\hline
\end{tabular}


is repeated replication -- assessing the evidence for the claim by independently regenerating evidence in multiple contexts. This can be applied focally for specific claims, usually at high cost. That is, it is highly accurate and not at all scalable. Each level from the bottom to the top of the table is presumed to offer increases in accuracy but with greater resource expenditure reducing its scalability. ${ }^{1}$

A first step for establishing a framework of credibility assessment metrics is to evaluate and validate existing methods. This will establish an evidence base for accuracy and resource cost of existing approaches, and inform prioritization of research investments to break linearity of costs and achieve scalability. The Figure below illustrates this goal. The $y$-axis represents accuracy achieved by the method, and the x-axis represents the resource costs for using the method. The diagonal trend represents a near-linear accuracy-cost tradeoff. In that circumstance, no method is preferable to another in terms of resource expense. For a fixed investment, the same amount of total information is gained conducting many replications of a single finding as conducting an automated assessment of many findings. Scalability is achieved by either lowering the resource demands of the methods, or by increasing the accuracy of methods requiring fewer resources per evaluated claim. Reducing resource demands is hard or impossible for many approaches because they have fixed costs. Increasing accuracy has substantial potential because there are promising ideas but little investment so far in advancing them, particularly for automated methods which also have the lowest resource requirements.

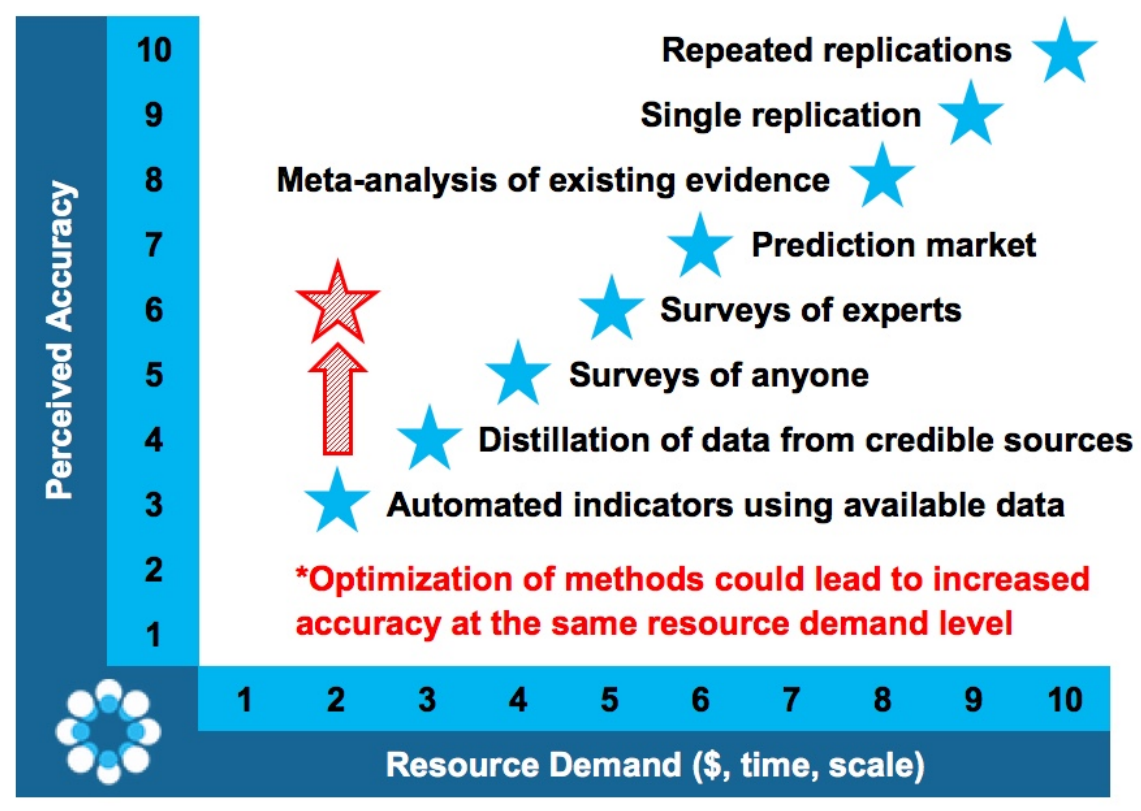

\footnotetext{
${ }^{1}$ One need not agree with the order of methods from lowest to highest. Indeed the goal of the work described herein is to acquire evidence about their relative accuracy, resource cost, and potential innovation to maximize the first and minimize the second.
} 
To achieve a baseline of evidence to inform further investment, we suggest that DARPA leverage a highly productive approach--a contest (Francescon 2017; Lai, Nosek et al. 2014). An open contest will identify and compare the qualities of existing State of the Art methods for assessing credibility and may also surface new ideas and technologies that are currently in development. Also, the contest will create and grow a community of researchers interested in these questions, from which an outstanding group of performers will likely emerge for a future DARPA program.

\section{The Challenge: Create the best performing metric for evaluating the credibility of research evidence}

\section{Entrants}

Anyone can enter the contest. Entrants can do whatever they want to create credibility indicators. Entrants must commit to preregistering their method on the Open Science Framework, sharing their output, and sharing their method by the end of the contest.

\section{Approach}

Organizers will provide entrants with "populations" of researchers, papers, or findings from which samples may be drawn for evaluation of the rankings. Entrants create evaluation criteria and apply them to the populations and output a ranking/rating of each item in the population. Organizers score the ranking against a sample of the population (the test set) that has one or more independent assessments of credibility. Entrants are scored on multiple criteria across multiple populations.

\section{Evaluation criteria}

The key evaluation criteria for each entry are:

- Accuracy - how well did the ranking correspond to independent ranking evidence of the test set

- Coverage - what portion of the population test was ranked by the method; what portion of the test set was ranked

- Efficiency - what was the resource cost of generating the rankings

Populations and independent credibility assessments

There is no gold standard for credibility assessment. As a consequence, deciding which method is working better than others requires iterative development of evidence for validity of a variety of indicators. Establishing validity of new constructs proceeds via the development of a nomological net (Cronbach \& Meehl, 1955) in which different sources provide mutually reinforcing evidence and iterative development and understanding of the construct. The contest will follow the strategy of nomological net 
development by using "populations" that correspond to test sets of a variety of sources of evidence for credibility of research evidence. Importantly, entrants will not know the test set in advance. They will only know the population and use that to prepare their ranking.

\begin{tabular}{|l|l|}
\hline Population (provided to Entrants) & $\begin{array}{l}\text { Test set criterion (method for scoring } \\
\text { entries) }\end{array}$ \\
\hline $\begin{array}{l}\text { Published papers in Psychology from } \\
2000 \text { to } 2015\end{array}$ & $\begin{array}{l}\text { Sample of papers with existing } \\
\text { replications from the population }\end{array}$ \\
\hline $\begin{array}{l}\text { Defined sample of papers from } \\
\text { Psychology and Economics }\end{array}$ & $\begin{array}{l}\text { Sample of papers with prediction market } \\
\text { data from these fields }\end{array}$ \\
\hline $\begin{array}{l}\text { Defined sample of papers from } \\
\text { Psychology, Political Science, and } \\
\text { Economics }\end{array}$ & $\begin{array}{l}\text { Papers that were assessed via surveys of } \\
\text { experts, with expert rated credibility as } \\
\text { the criterion }\end{array}$ \\
\hline $\begin{array}{l}\text { Defined sample of psychology } \\
\text { researchers }\end{array}$ & $\begin{array}{l}\text { Sample of those psychologists that were } \\
\text { rated by other psychologists for the } \\
\text { credibility of their research }\end{array}$ \\
\hline
\end{tabular}

Ex post facto evaluations of entry-generated rankings

In addition to the criteria above, independent experts will be provided the rankings of papers and researchers generated by the entries and evaluate the quality of the entries by evaluation of the rankings that they produced.

\section{Exploratory aggregated algorithms}

In all likelihood, a combination of assessment methods will yield a greater perceived accuracy than that of the methods individually. Exploratory aggregation of multiple methods, aided by the newly developed nomological net, will investigate the mathematical nature of these relationship and what the "optimal" combination of methods would be to either achieve a certain level of accuracy, or work within specific budget constraints. As a hypothetical example, combining the distillation of data from credible sources (accuracy $=4$ ) with surveys of anyone (accuracy $=5$ ) in a simple, linear fashion would yield an accuracy level of 9 , which is equal to a single replication. Considering the resource demand parameter, a single replication is designated as level 9 , however, the combination of data distillation (resource $=3$ ) and surveys of anyone (resource $=4$ ) would yield a resource demand of only 7 . In this example, the combination of two lower cost methods would yield the same accuracy as a single replication, but at a savings to overall resource demand. 
One could imagine a number of "optimized" algorithms arising from this exploration, with various assessment methods being weighted differently. Future work could be to identify a number of promising algorithms to test in confirmatory studies and investigate these in a variety of contexts. Through optimization and validation of these algorithms, the most influential assessment tools could be identified as potential future investments with the highest return on investment.

[What COS would contribute]

- Organize the contest as a metascience project

- Build a community of participants in the contest and promote the outcomes more broadly

- Guide entrants in the process of preregistration, sharing methods and data, using the OSF

- Analyze the rankings, determine winners, and conduct research developing the nomological net across these various indicators

- Exploratory analysis on the aggregation of various metrics to improve the accuracy of credibility assessment or decrease resource demand 


\section{$\underline{\text { References }}$}

Cronbach, L. J. \& Meehl, P. E. (1955) Construct Validity in Psychological Tests.

Psychological Bulletin, 52, 281-302. doi:10.1037/h0040957

Francescon, D. (2017) Contest aims to boost innovation in sustainability research. https://www.elsevier.com/connect/contest-aims-to-boost-innovation-in-sustainabilityresearch

Lai, C. K., Marini, M., Lehr, S. A., Cerruti, C., Shin, J. L., Joy-Gaba, J. A., Ho, A. K., Teachman, B. A., Wojcik, S. P., Koleva, S. P., Frazier, R. S., Heiphetz, L., Chen, E., Turner, R. N., Haidt, J., Kesebir, S., Hawkins, C. B., Schaefer, H. S., Rubichi, S., Sartori, G., Dial, C., Sriram, N., Banaji, M. R., \& Nosek, B. A. (2014). A comparative investigation of 17 interventions to reduce implicit racial preferences. Journal of Experimental Psychology: General, 143, 1765-1785. doi:10.1037/a0036260 